\title{
Erratum to: Green tea extract enhances parieto-frontal connectivity during working memory processing
}

\author{
André Schmidt • Felix Hammann • Bettina Wölnerhanssen • \\ Anne Christin Meyer-Gerspach • Jürgen Drewe • \\ Christoph Beglinger $\cdot$ Stefan Borgwardt
}

Published online: 4 July 2014

(C) Springer-Verlag Berlin Heidelberg 2014

\section{Erratum to: Psychopharmacology \\ DOI 10.1007/s00213-014-3526-1}

The original version of this article inadvertently contained a mistake. There are incorrect information found under Abstract and Experimental Design Sections. Please see below for the corrections.

1) Current version:

Abstract: Using a double-blind, counterbalanced, withinsubject design, 12 healthy volunteers received a milk wheybased soft drink containing $27.5 \mathrm{~g}$ of green tea extract or a milk whey-based soft drink without green tea as control substance while undergoing functional magnetic resonance imaging.

Should read:

Abstract: Using a double-blind, counterbalanced, within-subject design, 12 healthy volunteers received a milk whey-based soft drink containing green tea extract corresponding to $2,75 \mathrm{~g} / 1$ green tea leaves or a milk whey-based soft drink without green tea as control substance while undergoing functional magnetic resonance imaging

2) Current version:

Experimental Design: Participants received either 250 or $500 \mathrm{ml}$ milk whey-based soft drink containing 13.75 and $27.5 \mathrm{~g}$ of green tea extract, respectively (Rivella, Rothrist, Switzerland)

Should read:

Experimental Design: Participants received either 250 or $500 \mathrm{ml}$ milk whey-based soft drink containing 125 and $250 \mathrm{mg}$ of green tea extract corresponding to 2,75 g/l green tea leaves, respectively (Rivella, Rothrist, Switzerland).

The online version of the original article can be found at http://dx.doi.org/ 10.1007/s00213-014-3526-1.

A. Schmidt $\cdot$ S. Borgwardt $(\bowtie)$

Department of Psychiatry (UPK), University of Basel, Wilhelm

Klein Str. 27, 4012 Basel, Switzerland

e-mail: Stefan.Borgwardt@usb.ch

A. Schmidt $\cdot$ S. Borgwardt

Medical Image Analysis Center, Schanzenstrasse 55, 4031 Basel,

Switzerland

F. Hammann • B. Wölnerhanssen · A. C. Meyer-Gerspach •

J. Drewe $\cdot \mathrm{C}$. Beglinger

Department of Gastroenterology, University Hospital Basel,

4031 Basel, Switzerland

S. Borgwardt

Department of Psychosis Studies, Institute of Psychiatry, King's

College London, London, UK 